OPEN

SUBJECT AREAS:

QUANTUM

INFORMATION

QUBITS

QUANTUM MECHANICS

Received

16 June 2014

Accepted

13 October 2014

Published

31 October 2014

Correspondence and requests for materials should be addressed to

G.-L.L. Igllong@ tsinghua.edu.cn)

\section{Experimental Optimal Single Qubit Purification in an NMR Quantum Information Processor}

\author{
Shi-Yao Hou ${ }^{1,2,3}$, Yu-Bo Sheng 1,4, Guan-Ru Feng ${ }^{1,2,3}$ \& Gui-Lu Long ${ }^{1,2,3}$
}

\author{
'State Key Laboratory of Low-dimensional Quantum Physics and Department of Physics, Tsinghua University, Beijing 100084, \\ China, ${ }^{2}$ The Innovative Center of Quantum Matter, Beijing 100084, China, ${ }^{3}$ Tsinghua National Laboratory of Information Science \\ and Technology, Beijing 100084, China, ${ }^{4}$ Institute of Signal Processing Transmission, Nanjing University of Posts and \\ Telecommunications, Nanjing, 210003, China.
}

High quality single qubits are the building blocks in quantum information processing. But they are vulnerable to environmental noise. To overcome noise, purification techniques, which generate qubits with higher purities from qubits with lower purities, have been proposed. Purifications have attracted much interest and been widely studied. However, the full experimental demonstration of an optimal single qubit purification protocol proposed by Cirac, Ekert and Macchiavello [Phys. Rev. Lett. 82, 4344 (1999), the CEM protocol] more than one and half decades ago, still remains an experimental challenge, as it requires more complicated networks and a higher level of precision controls. In this work, we design an experiment scheme that realizes the CEM protocol with explicit symmetrization of the wave functions. The purification scheme was successfully implemented in a nuclear magnetic resonance quantum information processor. The experiment fully demonstrated the purification protocol, and showed that it is an effective way of protecting qubits against errors and decoherence.
$\mathrm{P}$ rotecting the qubits' coherence against environmental noise is of vital importance. Generally, there are several different methods to protect qubits against noise, such as dynamical decoupling ${ }^{1-3}$, decoherence free subspace ${ }^{4,5}$, quantum error correction ${ }^{6,7}$ and purification protocols ${ }^{8-10}$. Quantum error correction works by redundantly encoding the quantum state into a higher-dimensional Hilbert space. Error syndromes, which are collective quantities, can be identified with measurement, and errors are then corrected by performing recovering gate operations. Purification technique obtains entangled states with higher purities from those with lower purities. These two methods have advantages in different situations. Compared to quantum error correction, purification protocols require less resources and simpler operations, and are better suited for cases where large quantities of simple quantum systems are needed, for instance, in quantum communications. Entanglement purification protocols with ${ }^{8,11,12}$, or without controlled-NOT (CNOT) gate ${ }^{9,13-15}$ were proposed. Moreover, purification of entanglement of different degrees of freedom ${ }^{16,17}$, and partially entangled photon pairs ${ }^{18,19}$ were put forward. Experimental realizations of entanglement purification schemes have also been reported ${ }^{10,20-22}$.

Cirac, Ekert and Macchiavello ${ }^{23}$ proposed the CEM protocol that purifies single qubits, and they showed that single qubits of better quality can be purified from two or more copies of degraded qubits. Single qubit states are the building blocks in quantum information processing, such as quantum teleportation ${ }^{24}$, quantum cryptography ${ }^{25}$, quantum computation ${ }^{26,27}$ and quantum metrology $y^{28}$. Therefore, the CEM protocol could be routinely used as a fundamental procedure in these tasks. The main idea of the CEM protocol is to use a set of projections onto a symmetric subspace of the input qubits. Suppose there are two input qubits. If a purification procedure succeeds, the state of the two qubits is projected onto a two-qubit symmetric subspace, formed by $|00\rangle$, $\left|\Psi_{+}\right\rangle=(|0\rangle|1\rangle+|1\rangle|0\rangle) / \sqrt{2}$ and $|11\rangle$, i.e. the triplet states. If the protocol fails, the two-qubit state is projected onto the antisymmetric subspace, formed by the singlet state $\left|\Psi_{-}\right\rangle=(|0\rangle|1\rangle-|1\rangle|0\rangle) / \sqrt{2}$.

In 2004, this protocol was first realized by Ricci et al. in a linear-optical system ${ }^{29}$. The experiment was based on the Hong-Ou-Mandel effect where a beam splitter scatters two encoded polarized photons into the same arm when they are projected onto the symmetric subspace. This experiment is a partial demonstration of the CEM protocol because the implementation in photon system cleverly took advantage of the bosonic nature of photons 
which exempted the symmetrization procedure in the CEM protocol. In general, physical systems are not necessarily bosonic, and the symmetrization procedure has to be realized explicitly. Full experimental realization of the CEM protocol, implementing the symmetrization explicitly, is demanding. The experimental challenge lies in the implementation of a complicated quantum network that involves the CNOT gates and the Toffoli gate, and demands a high level of precise quantum coherent controls. In this work, we designed an experiment scheme that implemented the complicated quantum network efficiently, and realized the universal purification of single qubits in a nuclear magnetic resonance (NMR) quantum information processor. Our experiment shows that the CEM protocol is an efficient way of obtaining high purity qubits.

\section{Results}

Now we briefly describe the theoretical model ${ }^{23}$. There is a group of single qubits. Due to noises, they are in mixed state of the form,

$$
\rho=\frac{1}{2}(1+\lambda \vec{n} \cdot \vec{\sigma})=c_{0}\left|0_{\vec{n}}\right\rangle\left\langle 0_{\vec{n}}\left|+c_{1}\right| 1_{\vec{n}}\right\rangle\left\langle 1_{\vec{n}}\right|,
$$

where $\vec{n}=\left(n_{x}, n_{y}, n_{z}\right)$ corresponds to a pure state $\left|0_{\vec{n}}\right\rangle=\alpha|0\rangle+\beta|1\rangle$, which is represented by a vector of unit length in the Bloch spheres as shown by the dashed arrows in Figure 1 . Here $c_{1}+c_{0}=1,\left|0_{\vec{n}}\right\rangle,\left|1_{\vec{n}}\right\rangle$ are the two eigenstates of $\vec{n} \cdot \vec{\sigma} .\left|1_{\vec{n}}\right\rangle=\beta^{*}|0\rangle-\alpha^{*}|1\rangle$, which is orthogonal to $\left|0_{\vec{n}}\right\rangle$ and denotes the error (Note that our definition is different from that of Ref. 23 where the role of state $\left|0_{\vec{n}}\right\rangle$ and $\left|1_{\vec{n}}\right\rangle$ is exchanged, namely $\left|0_{\vec{n}}\right\rangle$ is the state to be purified). The threedimensional vector $\vec{n}$ and the pure state $\left|0_{\vec{n}}\right\rangle$ are related by $\left(n_{x}=\right.$ $\left.\alpha^{*} \beta+\alpha \beta^{*}, n_{y}=-\alpha^{*} \beta+\alpha \beta^{*}, n_{z}=|\alpha|^{2}-|\beta|^{2}\right)$. Here $\lambda=c_{0}-c_{1}$ is the purity of the state, since if $\lambda=1,\left.\rho\right|_{\lambda=1}=(1+\vec{n} \cdot \vec{\sigma}) / 2=\left|0_{\vec{n}}\right\rangle\left\langle 0_{\vec{n}}\right|$ is a pure state, while if $\lambda=0,\left.\rho\right|_{\lambda=0}=I / 2$ is a maximumly mixed state.

Given a density matrix $\rho$, the fidelity $f(\rho)$ is defined as

$$
f(\rho)=\left\langle 0_{\vec{n}}|\rho| 0_{\vec{n}}\right\rangle .
$$

Suppose $\rho=(1+\lambda \vec{n} \cdot \vec{\sigma}) / 2$, the fidelity

$$
f(\rho)=\frac{\lambda+1}{2} .
$$

Let us consider the system with two input qubits, the density operator of the two qubits can be decomposed into the symmetric (triplet) and antisymmetric (singlet) subspace as follows,

$$
\begin{aligned}
\rho^{\otimes 2}= & c_{0} c_{1}\left|\Psi_{-\vec{n}}\right\rangle\left\langle\Psi_{-\vec{n}}\right|+\left(c_{0}^{2}\left|0_{\vec{n}} 0_{\vec{n}}\right\rangle\left\langle 0_{\vec{n}} 0_{\vec{n}}\right|\right. \\
& \left.+c_{0} c_{1}\left|\Psi_{+\vec{n}}\right\rangle\left\langle\Psi_{+\vec{n}}\left|+c_{1}^{2}\right| 1_{\vec{n}} 1_{\vec{n}}\right\rangle\left\langle 1_{\vec{n}} 1_{\vec{n}}\right|\right) .
\end{aligned}
$$

If the purification is successful, the density operator can be written as

$$
\begin{aligned}
\rho_{\text {out }}= & \frac{1}{c_{0}^{2}+c_{0} c_{1}+c_{1}^{2}}\left(c_{0}^{2}\left|0_{\vec{n}} 0_{\vec{n}}\right\rangle\left\langle 0_{\vec{n}} 0_{\vec{n}}\right|\right. \\
& +c_{0} c_{1}\left|\Psi_{+\vec{n}}\right\rangle\left\langle\Psi_{+\vec{n}}\left|+c_{1}^{2}\right| 1_{\vec{n}} 1_{\vec{n}}\right\rangle\left\langle 1_{\vec{n}} 1_{\vec{n}}\right|,
\end{aligned}
$$

with $1 /\left(c_{0}^{2}+c_{0} c_{1}+c_{1}^{2}\right)$ being the normalization constant. $\left|\Psi_{+\vec{n}}\right\rangle$ is the symmetric state in the basis $\left\{\left|0_{\vec{n}}\right\rangle,\left\langle 1_{\vec{n}}\right|\right\}$. Then the output reduced density matrix of either qubit is

$$
\begin{aligned}
\rho_{\text {out }}^{a}= & \rho_{\text {out }}^{b}=\frac{1}{c_{0}^{2}+c_{0} c_{1}+c_{1}^{2}}\left[\left(c_{0}^{2}+\frac{c_{0} c_{1}}{2}\right)\left|0_{\vec{n}}\right\rangle\left\langle 0_{\vec{n}}\right|\right. \\
& \left.+\left(c_{1}^{2}+\frac{c_{0} c_{1}}{2}\right)\left|1_{\vec{n}}\right\rangle\left\langle 1_{\vec{n}}\right|\right]=\frac{1}{2}\left(1+\lambda^{\prime} \vec{n} \cdot \vec{\sigma}\right),
\end{aligned}
$$

where $\lambda^{\prime}=\left(c_{0}^{2}-c_{1}^{2}\right) /\left(c_{0}^{2}+c_{0} c_{1}+c_{1}^{2}\right)$. Define the purification factor $F(\lambda)=\lambda^{\prime} / \lambda$, then

$$
F(\lambda)=\frac{4}{3+\lambda^{2}}
$$

It is clear that $F(\lambda)$ is always greater than 1 and approaches 1 as $\lambda \rightarrow 1$.

The procedures of the CEM protocol is shown in Figure 1. The circuit was given by Sciarrino et al. in Ref. 30. An ancillary qubit is initially prepared in state $|0\rangle\langle 0|$, and the input state of the two work qubits is a mixed state. After performing the Hadamard, CNOT and the Toffoli gate operations on the initial three-qubit system, the ancillary qubit will be conditionally flipped. If the state of qubit $a$ and qubit $b$ is $\left|\Psi_{-\vec{n}}\right\rangle\left\langle\Psi_{-\vec{n}}\right|$, which carries no information about the initial state, the ancillary qubit will flip and the two work qubits will be discarded. Otherwise, the ancillary qubit will not change and qubit $a$ and $b$ are kept. Therefore, by measuring the ancillary qubit, one can decide whether or not the purification is successful. Then the state can be recovered by performing a Hadamard and a CNOT operations.

In the experiment, different input states have been prepared. Let the initial states be $\rho=(1+\lambda \vec{n} \cdot \vec{\sigma}) / 2$. We prepared states with different purity parameters $\lambda$, which corresponds to states with different degrees of degradation. Three unit Bloch vectors, $\vec{n}_{1}=$ $(0,0,1), \vec{n}_{2}=(0,1,0)$, and $\vec{n}_{3}=(0, \sin (7 \pi / 20), \cos (7 \pi / 20))$, which correspond to $\left|0_{\vec{n}_{1}}\right\rangle=|0\rangle, \quad\left|0_{\vec{n}_{2}}\right\rangle=(|0\rangle+|1\rangle) / \sqrt{2}$ and $\left|0_{\vec{n}_{3}}\right\rangle=$ $\exp \left(-i 7 \pi \sigma_{x} / 40\right)|0\rangle$, were chosen respectively. In the NMR experimental demonstration, a pseudo pure state $|0\rangle$ is prepared at first. To prepare mixed states with different $\lambda$ 's, the pseudo-pure state $|0\rangle$ is rotated around the $x$-axis with angle $\theta$ and then followed by performing the gradient field. Hence the purity parameter has a simple expression in terms of $\theta, \lambda=\cos (\theta)$. This procedure is illustrated in Figure 2 and described in detail in Methods section. The $\theta$ angles were set to $\theta=\epsilon \times \frac{\pi}{2}$ with $\epsilon$ ranging from 0.1 to 0.9 with an interval of

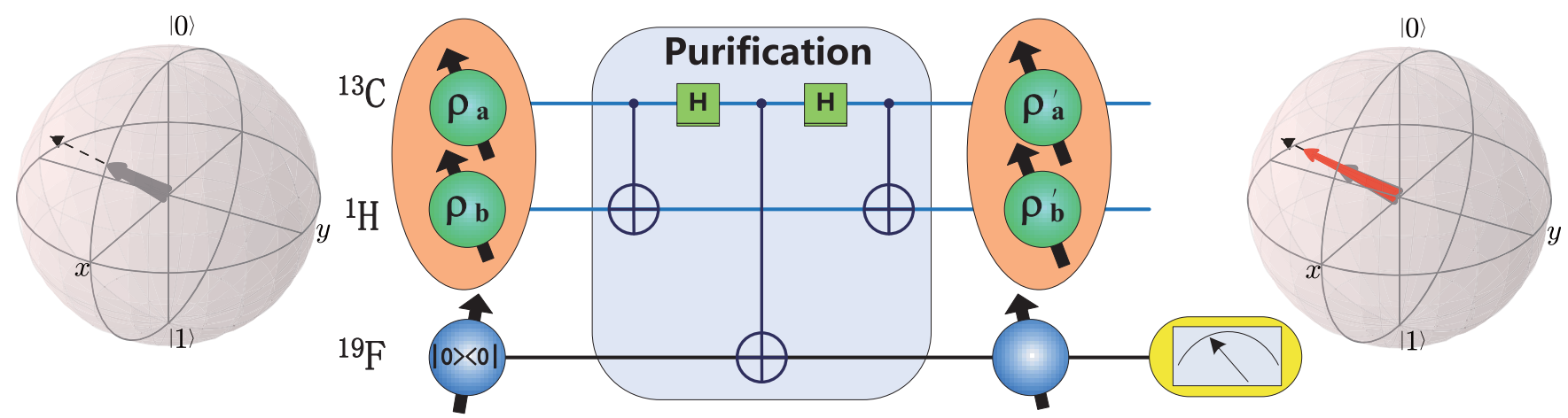

Figure $1 \mid$ The experimental scheme and the CEM circuit. The CEM circuit was given in Ref. 30. The input state of two work qubit is $\rho^{\otimes 2}=\rho_{a} \otimes \rho_{b}$, where $\rho$ is shown by a gray vector at the left Bloch sphere. The ancillary qubit is initially prepared at $|0\rangle\langle 0|$. After purification, the state of work qubits becomes $\rho^{\prime} \otimes \rho^{\prime}$, where $\rho^{\prime}$ is shown by a longer red arrow at the right Bloch sphere. The dashed arrow represents the pure state which is the target state of the purification. 
a)

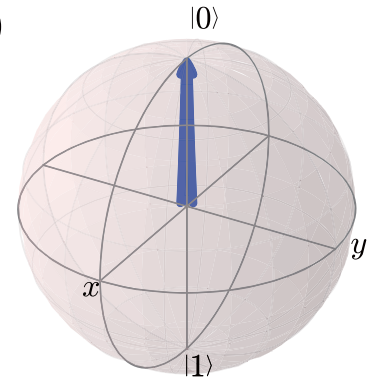

b)

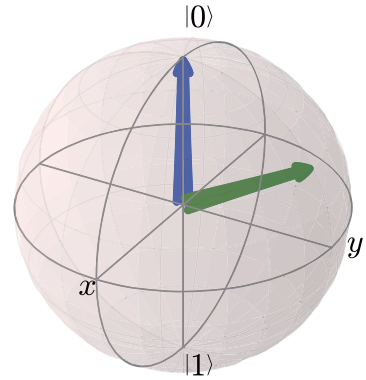

c)

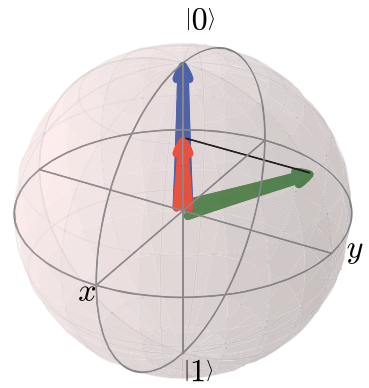

Figure 2| Procedure of input mixed state preparation: (a) pseudo-pure state $|0\rangle$ indicated by a blue vector along $z$-axis; (b) rotating the pseudo pure state through angle $\theta$ around the $x$ axis, shown by a green vector; (c) performing a gradient field to produce a mixed state shown by a short red vector.

0.1. The normalized density matrix of the output state is written as $\left(1+\lambda^{\prime} \vec{n} \cdot \vec{\sigma}\right) / 2$. It is clear that the purification factor $F(\lambda)$ is greater than 1 .

Full tomography process of whole three-qubit system is carried out first, and then the tomography of the output qubit is picked by tracing out the other two qubits. Because the sample is of natural abundance, the splitting in hydrogen and fluorine spectra caused by carbon could hardly be observed. To obtain their states, a
SWAP gate (which is also realized through radio-frequency pulses) is applied to transfer the state of hydrogen or fluorine to carbon, and be observed through the carbon spectrum. One example tomography result of the input and output state is shown in Figure 3. For the example, the initial purity is $\lambda=\cos (7 \pi / 20)=$ 0.454 , which means the initial state is $\rho_{i}=\left(1+0.454 \sigma_{z}\right) / 2$. The purity of the output state is $\lambda^{\prime}=0.566$, which is larger than the input state, giving $F(\lambda)=1.25$.

\section{Theory}

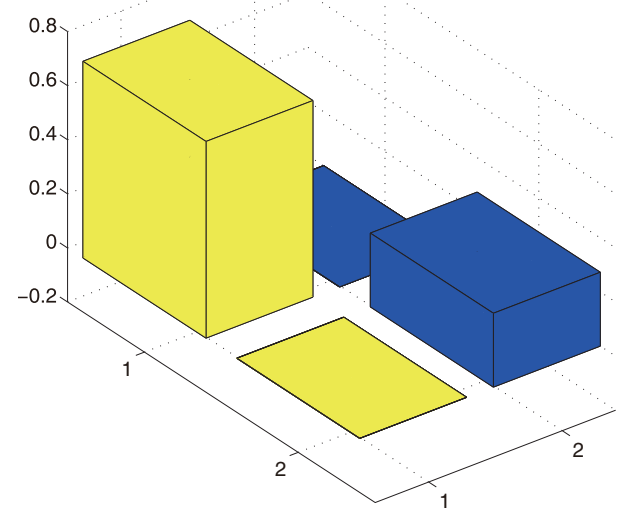

a)

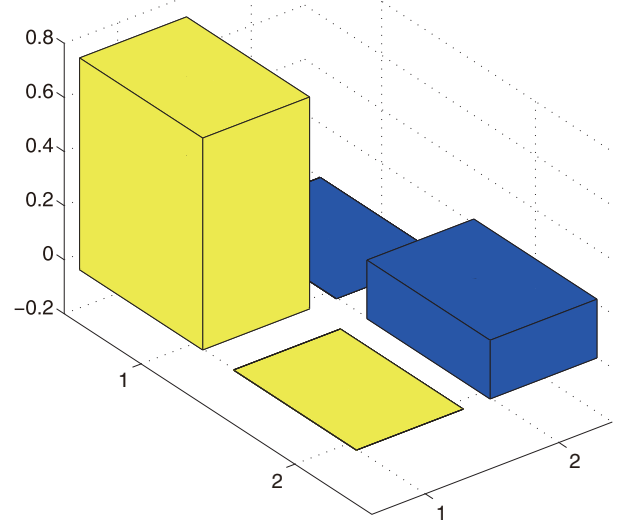

c)

\section{Experiment}

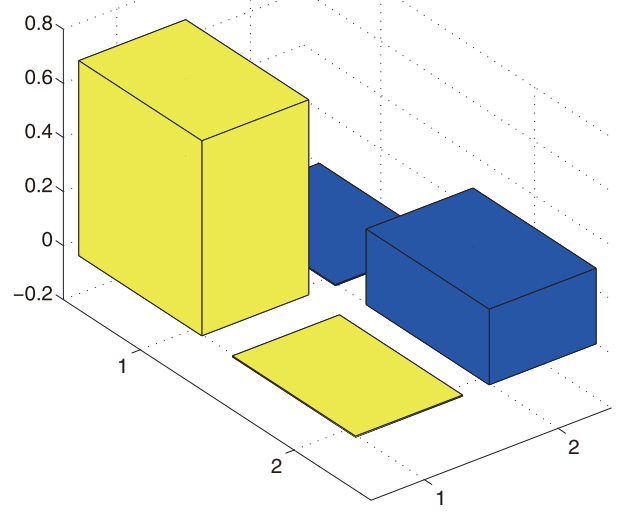

b)

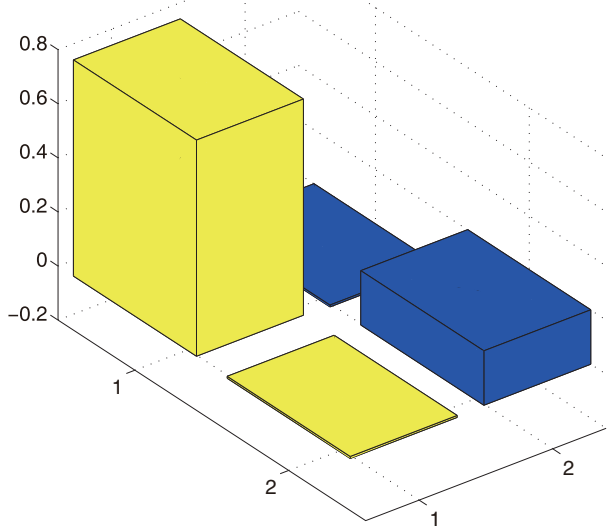

d)

Figure $3 \mid$ Comparison of the density matrices with $\lambda=\cos (7 \pi / 20)$ : theoretical (a) and experimental (b) input density matrix with $\rho_{\text {in }}=\left(I+\lambda \sigma_{z}\right)$, and theoretical (c) and experimental (d) output density matrix with $\rho_{\text {out }}=\left(I+\lambda^{\prime} \sigma_{z}\right)$, where $\lambda^{\prime}=4 \lambda /\left(3+\lambda^{2}\right)=0.5664$. 
Theoretical and experimental results of purification factors and the state fidelities after purification are compared in Figure 4. In Figures 4a), $4 b$ ) and 4c), the experimental result of purification factors are compared with the theoretical results for states $\left|0_{\vec{n}_{1}}\right\rangle=|0\rangle,\left|0_{\vec{n}_{2}}\right\rangle=(|0\rangle+|1\rangle) / \sqrt{2}$ and $\left|0_{\vec{n}_{3}}\right\rangle=\exp \left(-i 7 \pi \sigma_{x} / 40\right)|0\rangle$, respectively. Each data point is obtained by repeating one the experiment for eight times, and the standard deviation is obtained and shown as the error bar. The experimental results agree with theoretical predictions very well.
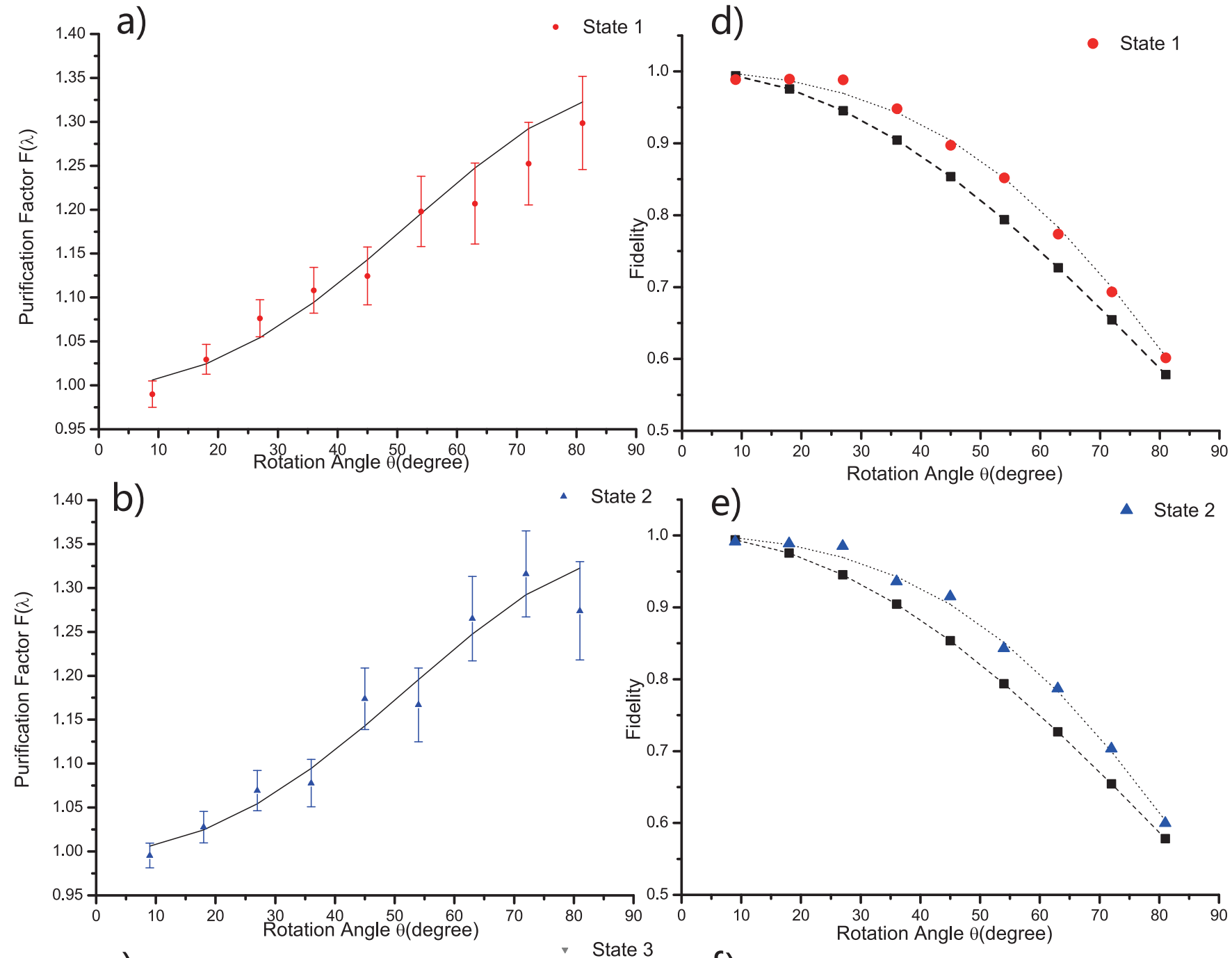

e)

$\Delta$ State 2
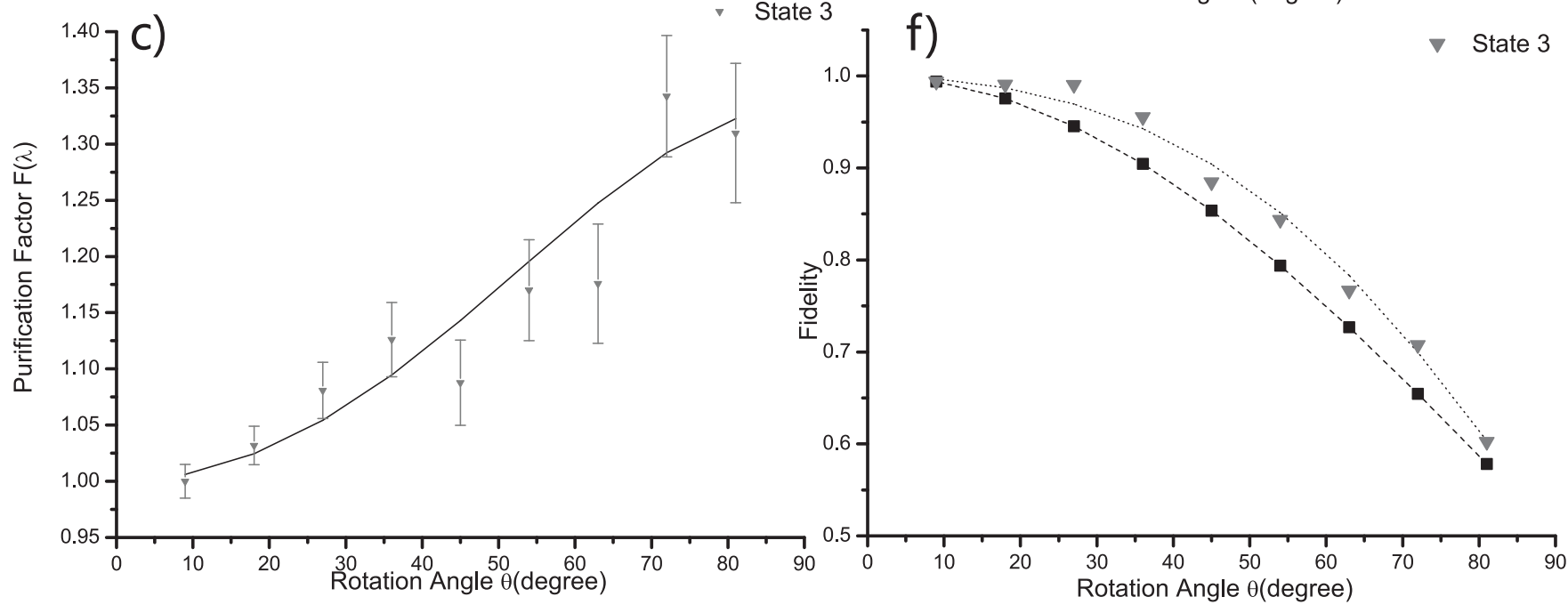

Figure $4 \mid$ Result of purification. State 1 (red), state 2 (blue) and state 3 (gray) refer to $|0\rangle,(|0\rangle+|1\rangle) / \sqrt{2}$ and $\exp \left(-i 7 \pi \sigma_{x} / 40\right)|0\rangle$ respectively. The rotation angles represent the purity $\lambda=\cos (\theta)$. Sub-figures (a), (b) and (c) are the purification factors obtained in the experiment. The experiments were repeated for eight times and the standard deviations were calculated as the error bar. Sub-figures (d), (e) and (f) show the fidelity before and after the purification. The black squares(triangles) represent the fidelities of the initial(output) pseudo mixed states. The dashed line and dotted line represent the theoretical fidelities before and after purification, respectively. 
In Figures $4 \mathrm{~d}$ ), 4e) and 4f), we plotted the fidelities of the input (as square in the figure) and output (as triangle in the figure) states compared with the theoretical value for states $\left|0_{\vec{n}_{1}}\right\rangle=|0\rangle$, $\left|0_{\vec{n}_{2}}\right\rangle=(|0\rangle+|1\rangle) / \sqrt{2}$ and $\left|0_{\vec{n}_{3}}\right\rangle=\exp \left(-i 7 \pi \sigma_{x} / 40\right)|0\rangle$, respectively. In Ref. 23 , the fidelity of the output state after purification is given explicitly. In the current situation, the fidelity after purification is $f\left(\rho_{\text {out }}\right)=c_{0}\left(1-c_{1} / 2\right) /\left(1-c_{0} c_{1}\right)>c_{0}$. The experimental data agree well with this theoretical prediction. The good agreement between experimental results and theoretical prediction for both the purification factors and the fidelities of the states after purification shows that the protocol is effective and universal.

In the experiments, the deviations of the experimental results from the theoretical predictions are mainly due to the inhomogeneities in the magnetic fields, the inaccuracy in the gate operations and decoherence due to noise. The low signal-to-noise ratio (SNR) also contributes to the deviation because the sample used in our experiment is of natural abundance, and the percentage of ${ }^{13} \mathrm{C}$ is only about $1 \%$. The low signal is more pronounced for states with a lower fidelity, because initial mixed state is prepared by applying a Z-gradient, and the Zgradient kills the transverse polarization and thus the signal decreases. Hence, the lower the purity of the initial state, the weaker the SNR. This explains why the error bars of the purification factors increase as the purities of the initial state decrease.

\section{Discussion}

It is interesting to compare the current CEM protocol with the traditional entanglement purification. Pan et al. discussed and realized the photonic polarization entanglement purification for bit-flip error $^{9,10}$. They showed that the bit-flip error can be well corrected by selecting the four-mode cases. In this way, the two cross-combinations, which cannot lead to the four-mode cases, are eliminated automatically. The cross-combinations essentially are the two pairs which only contain one bit-flip error. In the CEM protocol, the original mixed states can also be described as a probabilistic mixture of four pure states: i) they are in the state $\left|0_{\vec{n}}\right\rangle \otimes\left|0_{\vec{n}}\right\rangle$, with a probability of $c_{0}^{2}$, ii) in the states $\left|0_{\vec{n}}\right\rangle \otimes\left|1_{\vec{n}}\right\rangle$ and $\left|1_{\vec{n}}\right\rangle \otimes\left|0_{\vec{n}}\right\rangle$, with equal probabilities of $c_{0} c_{1}$, and iii) in the state $\left|1_{\vec{n}}\right\rangle \otimes\left|1_{\vec{n}}\right\rangle$, with a probability of $c_{1}^{2}$. In detail, the four states could be expanded as follows,

$$
\begin{gathered}
\left|0_{\vec{n}}\right\rangle \otimes\left|0_{\vec{n}}\right\rangle=(\alpha|0\rangle+\beta|1\rangle) \otimes(\alpha|0\rangle+\beta|1\rangle) \\
=\alpha^{2}|00\rangle+\sqrt{2} \alpha \beta\left|\Psi_{+}\right\rangle+\beta^{2}|11\rangle, \\
\left|0_{\vec{n}}\right\rangle \otimes\left|1_{\vec{n}}\right\rangle=(\alpha|0\rangle+\beta|1\rangle) \otimes\left(\beta^{*}|0\rangle-\alpha^{*}|1\rangle\right) \\
=\alpha \beta^{*}|00\rangle-\frac{|\beta|^{2}-|\alpha|^{2}}{\sqrt{2}}\left|\Psi_{+}\right\rangle-\beta \alpha^{*}|11\rangle-\frac{1}{\sqrt{2}}\left|\Psi_{-}\right\rangle,
\end{gathered}
$$

$$
\begin{aligned}
\left|1_{\vec{n}}\right\rangle \otimes\left|0_{\vec{n}}\right\rangle & =\left(\beta^{*}|0\rangle-\alpha^{*}|1\rangle\right) \otimes(\alpha|0\rangle+\beta|1\rangle) \\
& =\beta^{*} \alpha|00\rangle+\frac{|\beta|^{2}-|\alpha|^{2}}{\sqrt{2}}\left|\Psi_{+}\right\rangle-\alpha^{*} \beta|11\rangle+\frac{1}{\sqrt{2}}\left|\Psi_{-}\right\rangle
\end{aligned}
$$

$$
\begin{aligned}
\left|1_{\vec{n}}\right\rangle \otimes\left|1_{\vec{n}}\right\rangle & =\left(\beta^{*}|0\rangle-\alpha^{*}|1\rangle\right) \otimes\left(\beta^{*}|0\rangle-\alpha^{*}|1\rangle\right) \\
& =\beta^{*^{2}}|00\rangle-\sqrt{2} \alpha^{*} \beta^{*}\left|\Psi_{+}\right\rangle+\alpha^{*^{2}}|11\rangle .
\end{aligned}
$$

Note that $\left|\Psi_{-\vec{n}}\right\rangle=\left(\left|0_{\vec{n}}\right\rangle\left|1_{\vec{n}}\right\rangle-\left|1_{\vec{n}}\right\rangle\left|0_{\vec{n}}\right\rangle\right) / \sqrt{2}=(|0\rangle|1\rangle-|1\rangle|0\rangle) /$ $\sqrt{2}$, is invariant with respect to the rotation of $\vec{n}$. From Eqs. (8)-(11), we cannot eliminate the cross-combination items $\left|0_{\vec{n}}\right\rangle \otimes\left|1_{\vec{n}}\right\rangle$ and $\left|1_{\vec{n}}\right\rangle \otimes\left|0_{\vec{n}}\right\rangle$ completely. Fortunately, we can partially eliminate the cross-combination items, for both cross-combination items contain the singlet state $\left|\Psi_{-}\right\rangle$. Therefore, by projecting the states onto the symmetric subspace, we can complete the purification task.
The CEM protocol focuses on unknown single qubits, which is the fundamental building blocks in many quantum communication and quantum computation protocols, which could hardly avoid preparing single qubits with high purities. The realization of CEM protocol in Ref. 29 took advantage of the Hong-Ou-Mandel effect. It is suitable for optical system, which applies to almost all quantum communication protocols. In this experiment, we fully demonstrated the CEM protocol in an NMR system. Different from the realization in linear optical system, we exploit the quantum circuit to project the initial mixed states onto the symmetric subspace. This experiment showed that the circuit protocol is a universal approach to purify the single qubit.

\section{Conclusion}

In conclusion, we have fully realized the CEM protocol in a threequbit NMR quantum information processor. We also explained theoretically that the CEM protocol is quite different from the traditional entanglement purification, for it can partially eliminate the cross-combinations, i. e. the singlet state. Our experimental results show that the purification factor agrees well with the theoretical prediction. This experiment fully demonstrated the original CEM protocol proposed by Cirac et al. As single qubits are the building blocks in quantum information processing, the CEM protocol is a powerful way for protecting single qubits from decoherence.

\section{Methods}

System and experiment procedure. The protocol is implemented on a BRUKER AVANCE III $400 \mathrm{MHz}$ NMR spectrometer with diethyl-fluoromalonate dissolved in $d 6$-acetone at $295.0 \mathrm{~K}$ as the quantum information processor. As shown in Figure 5, qubit $a$, qubit $b$ and the ancillary qubit are represented by ${ }^{1} \mathrm{H},{ }^{13} \mathrm{C}$, and ${ }^{19} \mathrm{~F}$, respectively. The natural Hamiltonian of these three nuclei of the molecule placed in a static magnetic field is

$$
H_{n a t}=\sum_{i} \omega_{i} \sigma_{z i}+\frac{\pi}{2} \sum_{i<j} J_{i j} \sigma_{z i} \sigma_{z j}
$$

where $\omega_{i}$ is the Larmor frequency of the $i$-th nuclei and $J_{i j}$ is the scalar coupling of spin $i$ and $j$. The molecular parameters, structure as well as the thermal spectrum of the ${ }^{13} \mathrm{C}$ are shown in Figure 5. Note that each of the four peaks represent the state of the other two qubits, and this helps us to decide the state of the ancillary qubit after the purification operation.

In our experiment, we realized the purification of three different input states:

$\left|0_{\vec{n}_{1}}\right\rangle=|0\rangle,\left|0_{\vec{n}_{2}}\right\rangle=(|0\rangle+|1\rangle) / \sqrt{2},\left|0_{\vec{n}_{3}}\right\rangle=\exp \left(-i \frac{7}{40} \pi \sigma_{x}\right)|0\rangle=0.8526|0\rangle-$ $0.5225 i|1\rangle$. The pulse sequence for the experiment is shown in Figure 6. First, the system should be initialized in a pure state, and for an ensemble system like NMR, a pseudo-pure state(PPS) is prepared. Second, to demonstrate the purification protocol, qubit $a$ and $b$ need be prepared in an mixed state. Similar to what we have done with pure state on an ensemble system, a pseudo mixed state is prepared. Third, after the preparation of the mixed state, the purification process is applied. After all these processes are completed, measurement is performed to obtain the result of the experiment. We now discuss these procedures in details as follows.

Initial state preparation. First of all, we create the pseudo pure state $\rho_{P P S}^{*}=\frac{(1-\epsilon)}{8} I+\epsilon|000\rangle\langle 000|$, where $\epsilon \sim 10^{-5}$ is the thermal polarization of the nuclear spin system, using the spatial averaging method ${ }^{31}$. The pulse sequence for preparing the state is shown in the PPS Preparation block in Figure 6.

Then what we want is the mixed state, which could be prepared using gradient fields ${ }^{32}$ or through the superposition of density matrices ${ }^{33}$. Here the method using gradient fields is employed. Apply a rotation of angle $\theta$ to qubit $a$ around the $x$-axis, which makes PPS become $\left(I+\cos (\theta) \sigma_{z}+\sin (\theta) \sigma_{y}\right)\left(I+\sigma_{z}\right)\left(I+\sigma_{z}\right)$. After performing a gradient field along the $z$-axis, the density matrix of qubit $a$ can be described as $I+\cos (\theta) \sigma_{z}$, which means a pseudo-mixed state on qubit $a$ is created. By applying the same procedure to qubit $b$, a pseudo-mixed state with density matrix

$$
\rho_{m}=\frac{1}{8}\left(I+\lambda \sigma_{z}\right)\left(I+\lambda \sigma_{z}\right)\left(I+\sigma_{z}\right)=\frac{1}{8}(1+\lambda \vec{k} \cdot \vec{\sigma})^{\otimes 2} \otimes|0\rangle\langle 0|
$$

is prepared, where $\lambda=\cos (\theta)$ and $\vec{k}$ is the unit vector along the $z$-axis. At last, to prepare an arbitrary mixed state $\rho_{\text {arb }}=\frac{1}{8}(1+\lambda \vec{n} \cdot \vec{\sigma})^{\otimes 2} \otimes|0\rangle\langle 0|$, a single qubit operation $U_{a}$, which can also be seen as a single qubit rotation that rotate $\vec{k}$ to any arbitrary direction $\vec{n}$, is applied to both qubits $a$ and $b$. The pulse sequence for pseudomixed state preparation can be seen in the corresponding block of Figure 6. 


\begin{tabular}{|c|c|c|c|c|c|}
\hline & ${ }^{13} \mathrm{C}$ & ${ }^{1} \mathrm{H}$ & ${ }^{19} \mathrm{~F}$ & T1 & T2 \\
\hline${ }^{13} \mathrm{C}$ & $2180.33 \mathrm{~Hz}$ & & & $3.13 \mathrm{~s}$ & $1.10 \mathrm{~s}$ \\
\hline${ }^{1} \mathrm{H}$ & $160.06 \mathrm{~Hz}$ & $9190.61 \mathrm{~Hz}$ & & $1.88 \mathrm{~s}$ & $0.78 \mathrm{~s}$ \\
\hline${ }^{19} \mathrm{~F}$ & $48.34 \mathrm{~Hz}$ & $-194.67 \mathrm{~Hz}$ & $-73324.05 \mathrm{~Hz}$ & $3.60 \mathrm{~s}$ & $1.18 \mathrm{~s}$ \\
\hline
\end{tabular}

a)

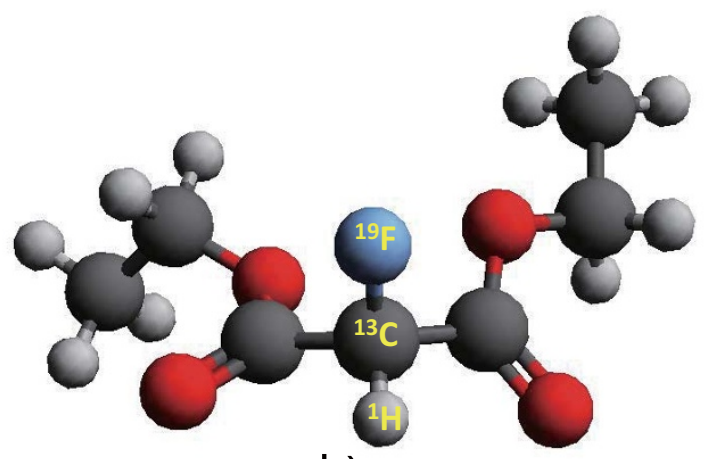

b)

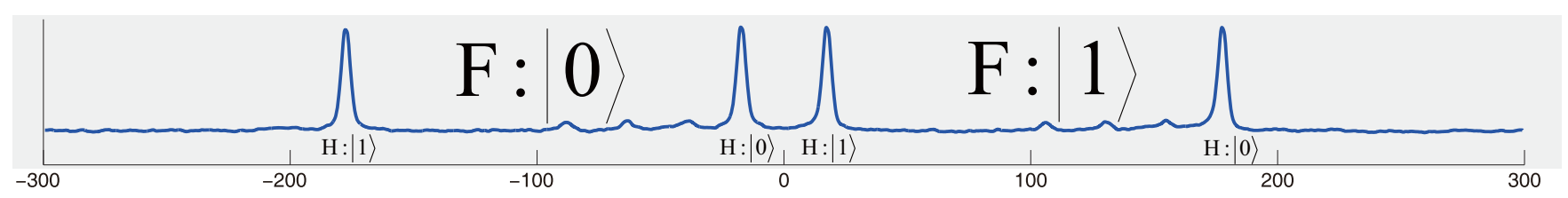

c)

Figure $5 \mid$ Molecular parameters, structure and the thermal spectrum of ${ }^{13} \mathrm{C}$ of diethyl-fluoromalonate. (a) The scalar couplings and chemical shifts of the three nuclei. (b) The molecular structure, where three nuclei used as the three qubits are marked. (c) The thermal equilibrium spectrum of the ${ }^{13} \mathrm{C}$ marked in the molecule.

Realization of purification process. After the mixed state is prepared, the purification protocol is applied. There are two approaches of implementing an arbitrary unitary operation on an NMR system. One is to decompose the unitary operations into one-qubit operations and two-qubit $\sigma_{z} \sigma_{z}$ interactions which can be implemented directly on an NMR spectrometer with radio frequency pulses and time delays ${ }^{34}$. The other is to use the optimal control technique to get an optimized shaped pulse with high fidelity to better control the system. One way of doing this is to use a GRAPE pulse which requires a full knowledge of the physical system ${ }^{35,36}$. The goal of this experiment is to demonstrate the effectiveness of the purification protocol and the pulse for this protocol can be regarded as a fundamental operation for future quantum information processing. Therefore, we adopt the GRAPE pulse. The pulse is found with a fidelity of 0.9995 and optimized so that it is robust to the fluctuations of the power of the spectrometer, the chemical shift of the molecules, and other parameters in the experiment ${ }^{37}$. The number of the steps of the pulse is $10^{3}$ and the duration of each step is $10 \mu \mathrm{s}$, so the total duration of the pulse is $10 \mathrm{~ms}$.

Result readout. The last step of the protocol is to measure the ancillary qubit: if the state of that qubit is $|0\rangle$, the protocol is successful and we keep the two work qubits. Otherwise, if the state of the qubit is $|1\rangle$, both work qubits are discarded. For simplicity, before measurement, we rotate $\vec{n}$ to $\vec{k}$ by applying $U_{a}^{\dagger}$ to both qubit $a$ and qubit $b$. Figure $5 c$ ) shows the spectra of ${ }^{13} \mathrm{C}$, which is assigned as qubit $a$. The four peaks of the spectrum from left to right represent that the nuclei ${ }^{1} \mathrm{H}$ and ${ }^{19} \mathrm{~F}$ are in the states $|10\rangle,|00\rangle,|11\rangle$, and $|01\rangle$, respectively. With respect to the state of ${ }^{19} \mathrm{~F}$, which is assigned as the ancillary qubit, the four peaks are divided into two groups: group 1 ,

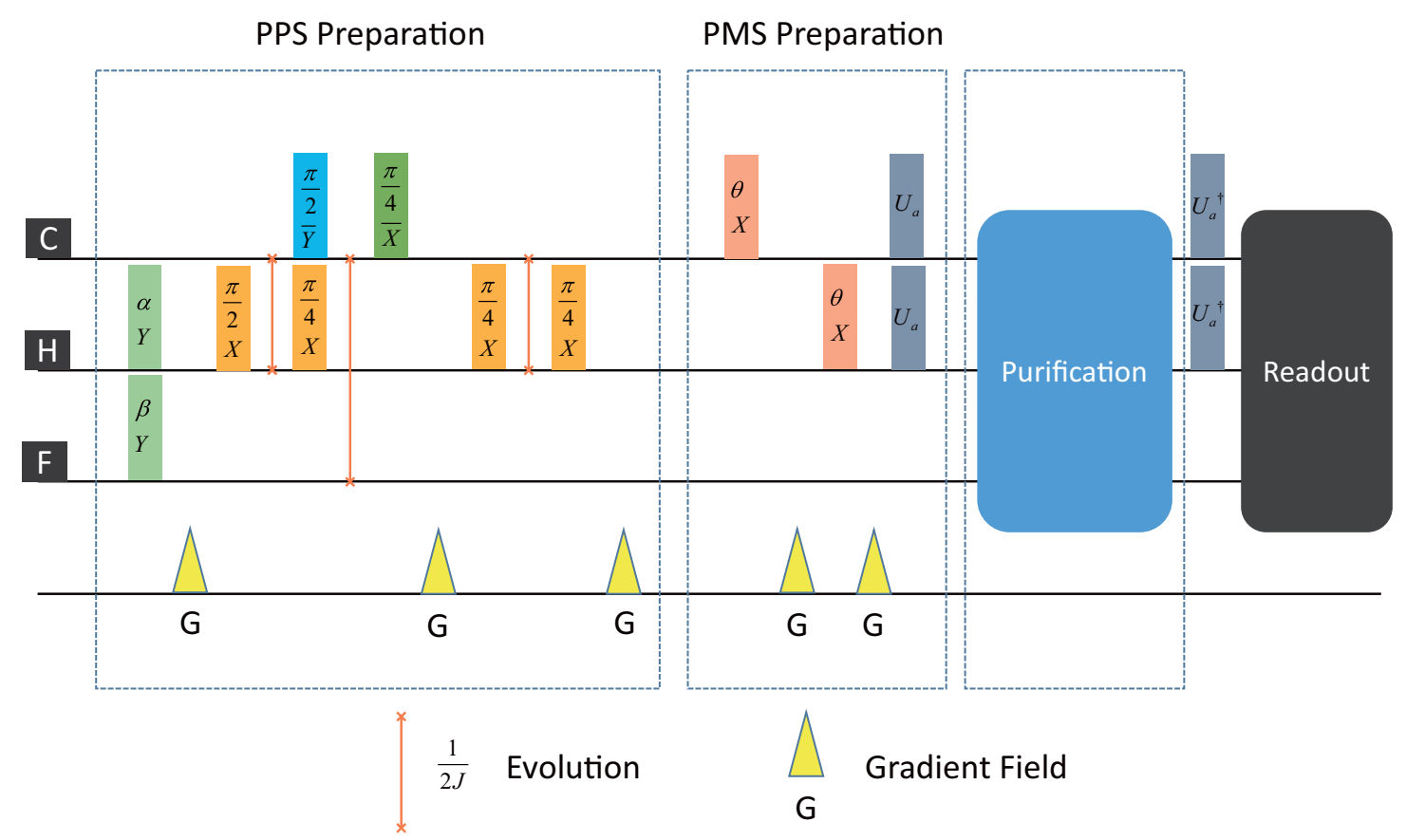

Figure $6 \mid$ Pulse sequence for the experiment. The small blocks refer to the single qubit rotations applied in NMR system, with the angle and axis of rotation shown inside the blocks. $U_{a}$ is applied to qubits $a$ and $b$ to create an arbitrary state that is to be purified. To simplify the readout process, after the purification process is completed, $U_{a}^{\dagger}$ is applied. 
consisting of the left two peaks, corresponds to the state $|0\rangle$ of ${ }^{19} \mathrm{~F}$, indicating that the purification process successful, and group 2, consisting of the right two peaks, corresponds to the state $|1\rangle$ of ${ }^{19} \mathrm{~F}$, indicating that the purification process failed. Let the intensity of the two peaks of the left two peaks after purification be denoted by $A_{1}$ and $A_{0}$, respectively. Then the purity $\lambda^{\prime}$ after purification is calculated as

$$
\lambda^{\prime}=\frac{A_{0}-A_{1}}{A_{0}+A_{1}} .
$$

With purity $\lambda^{\prime}$ the purification factor $F(\lambda)=\lambda^{\prime} / \lambda$ and the fidelity of output density matrix $f\left(\rho_{\text {out }}\right)$ can be obtained and compared to theoretical prediction. To better demonstrate the result, each experiment was repeated for eight times and the standard deviation of the purification factors were determined and is used as the error bar, shown in Figure 4

1. Yang, W., Wang, Z.-Y. \& Liu, R.-B. Preserving qubit coherence by dynamical decoupling. Front. Phys. 6, 2-14 (2011).

2. Souza, A. M., Álvarez, G. A. \& Suter, D. Robust dynamical decoupling. Phil. Trans. R. Soc. A 370, 4748-4769 (2012).

3. $\mathrm{Du}$, J. et al. Preserving electron spin coherence in solids by optimal dynamical decoupling. Nature 461, 1265-1268 (2009).

4. Lidar, D. A. Review of decoherence free subspaces, noiseless subsystems, and dynamical decoupling. Quantum Information and Computation for Chemistry: Advances in Chemical Physics. 154, 295-354 (2012).

5. Lidar, D. \& Birgitta Whaley, K. Decoherence-free subspaces and subsystems. In Benatti, F. \& Floreanini, R. (eds.) Irreversible Quantum Dynamics, Lecture Notes in Physics. Vol. 622, 83-120 (Springer Berlin Heidelberg, 2003).

6. Shor, P. W. Scheme for reducing decoherence in quantum computer memory. Phys. Rev. A 52, R2493-R2496 (1995).

7. Steane, A. M. Error correcting codes in quantum theory. Phys. Rev. Lett. 77, 793-797 (1996).

8. Bennett, C. H. et al. Purification of noisy entanglement and faithful teleportation via noisy channels. Phys. Rev. Lett. 76, 722-725 (1996).

9. Pan, J.-W., Simon, C., Brukner, C. \& Zeilinger, A. Entanglement purification for quantum communication. Nature 410, 1067-1070 (2001)

10. Pan, J.-W., Gasparoni, S., Ursin, R., Weihs, G. \& Zeilinger, A. Experimental entanglement purification of arbitrary unknown states. Nature 423, 417-422 (2003).

11. Deutsch, D. et al. Quantum privacy amplification and the security of quantum cryptography over noisy channels. Phys. Rev. Lett. 77, 2818-2821 (1996)

12. Duan, L.-M., Giedke, G., Cirac, J. I. \& Zoller, P. Entanglement purification of Gaussian continuous variable quantum states. Phys. Rev. Lett. 84, 4002-4005 (2000).

13. Gonţa, D. \& van Loock, P. Dynamical entanglement purification using chains of atoms and optical cavities. Phys. Rev. A 84, 042303 (2011)

14. Bennett, C. H., Bernstein, H. J., Popescu, S. \& Schumacher, B. Concentrating partial entanglement by local operations. Phys. Rev. A 53, 2046-2052 (1996).

15. Zhao, Z., Pan, J.-W. \& Zhan, M. S. Practical scheme for entanglement concentration. Phys. Rev. A 64, 014301 (2001).

16. Simon, C. \& Pan, J.-W. Polarization entanglement purification using spatial entanglement. Phys. Rev. Lett. 89, 257901 (2002).

17. Sheng, Y.-B., Zhou, L. \& Long, G.-L. Hybrid entanglement purification for quantum repeaters. Phys. Rev. A 88, 022302 (2013).

18. Yamamoto, T., Koashi, M. \& Imoto, N. Concentration and purification scheme for two partially entangled photon pairs. Phys. Rev. A 64, 012304 (2001).

19. Sheng, Y.-B., Zhou, L., Zhao, S.-M. \& Zheng, B.-Y. Efficient single-photon-assisted entanglement concentration for partially entangled photon pairs. Phys. Rev. A 85, 012307 (2012).

20. Salart, D. et al. Purification of single-photon entanglement. Phys. Rev. Lett. 104, 180504 (2010)

21. Zu, C. et al. Experimental distillation of quantum nonlocality. Phys. Rev. Lett. 111 , 050405 (2013)

22. Hage, B. et al. Preparation of distilled and purified continuous-variable entangled states. Nat. Phys. 4, 915-918 (2008)

23. Cirac, J. I., Ekert, A. K. \& Macchiavello, C. Optimal purification of single qubits. Phys. Rev. Lett. 82, 4344-4347 (1999).
24. Bennett, C. H. et al. Teleporting an unknown quantum state via dual classical and Einstein-Podolsky-Rosen channels. Phys. Rev. Lett. 70, 1895-1899 (1993).

25. Bennett, C. H., Brassard, G. et al. Quantum cryptography: Public key distribution and coin tossing. In Proceedings of IEEE International Conference on Computers, Systems and Signal Processing, Vol. 175 (New York, 1984).

26. Knill, E., Laflamme, R. \& Milburn, G. J. A scheme for efficient quantum computation with linear optics. Nature 409, 46-52 (2001).

27. Bassett, L. C. \& Awschalom, D. D. Quantum computation: Spinning towards scalable circuits. Nature 489, 505-507 (2012).

28. Giovannetti, V., Lloyd, S. \& Maccone, L. Quantum metrology. Phys. Rev. Lett. 96 , 010401 (2006).

29. Ricci, M. et al. Experimental purification of single qubits. Phys. Rev. Lett. 93 , 170501 (2004).

30. Sciarrino, F., Sias, C., Ricci, M. \& Martini, F. D. Quantum cloning and universal NOT gate by teleportation. Phys. Lett. A 323, 34-39 (2004).

31. Cory, D. G., Price, M. D. \& Havel, T. F. Nuclear magnetic resonance spectroscopy: An experimentally accessible paradigm for quantum computing. Physica $D$ : Nonlinear Phenomena 120, 82-101 (1998).

32. Du, J. et al. Observation of geometric phases for mixed states using NMR interferometry. Phys. Rev. Lett. 91, 100403 (2003).

33. Tang, Y.-C., Li, Y.-S., Hao, L., Hou, S.-Y. \& Long, G. L. Quantum-nondemolition determination of an unknown werner state. Phys. Rev. A 85, 022329 (2012).

34. Vandersypen, L. M. K. \& Chuang, I. L. NMR techniques for quantum control and computation. Rev. Mod. Phys. 76, 1037-1069 (2005).

35. Khaneja, N., Reiss, T., Kehlet, C., Schulte-Herbrüggen, T. \& Glaser, S. J. Optimal control of coupled spin dynamics: design of NMR pulse sequences by gradient ascent algorithms. J. Magn. Reson. 172, 296-305 (2005).

36. Schulte-Herbrüggen, T., Spörl, A., Khaneja, N. \& Glaser, S. J. Optimal controlbased efficient synthesis of building blocks of quantum algorithms: A perspective from network complexity towards time complexity. Phys. Rev. A 72, 042331 (2005).

37. Ryan, C. A., Negrevergne, C., Laforest, M., Knill, E. \& Laflamme, R. Liquid-state nuclear magnetic resonance as a testbed for developing quantum control methods. Phys. Rev. A 78, 012328 (2008).

\section{Acknowledgments}

This work is supported by the National Natural Science Foundation of China under Grants No.11474181 and No.11474168, the National Basic Research Program of China under Grant No. 2011CB9216002 and the Open Research Fund Program of the State Key Laboratory of Low-Dimensional Quantum Physics, Tsinghua University. We appreciate IQC, University of Waterloo, for providing the software package for NMR pulse optimization and simulation.

\section{Author contributions}

S.Y.H. and G.R.F. performed the experiments, S.Y.H., Y.B.S. and G.L.L. designed the experiment schemes; G.L.L. conceived the ideas and supervised the experiment. All authors contributed to the writing of the paper, discussed the theoretical protocol, experiment scheme and results.

\section{Additional information}

Competing financial interests: The authors declare no competing financial interests.

How to cite this article: Hou, S.-Y., Sheng, Y.-B., Feng, G.-R. \& Long, G.-L. Experimental Optimal Single Qubit Purification in an NMR Quantum Information Processor. Sci. Rep. 4, 6857; DOI:10.1038/srep06857 (2014).

This work is licensed under a Creative Commons Attribution-NonCommercialNoDerivs 4.0 International License. The images or other third party material in this article are included in the article's Creative Commons license, unless indicated otherwise in the credit line; if the material is not included under the Creative Commons license, users will need to obtain permission from the license holder in order to reproduce the material. To view a copy of this license, visit http:// creativecommons.org/licenses/by-nc-nd/4.0/ 